\title{
COMPLEMENTING CBT FOR DEPRESSED ADOLESCENTS WITH LEARNING THROUGH IN VIVO EXPERIENCE (LIVE): CONCEPTUAL ANALYSIS, TREATMENT DESCRIPTION, AND FEASIBILITY STUDY
}

\author{
Scott T. Gaynor and P. Scott Lawrence \\ University of North Carolina at Greensboro, USA
}

\begin{abstract}
Based on a behavior-analytic interpretation of the skills acquisition process, a rationale is presented for augmenting a currently available cognitive-behavioral treatment (The Adolescent Coping With Depression course: CWD-A) with a newly developed therapy focusing on the interpersonal interactions and learning occurring in-session (Learning through In-Vivo Experience: LIVE). A description of the practice of LIVE is offered and the results from an initial feasibility study are presented. Using a single-subject methodology, two groups each consisting of 5 adolescents with significant symptoms of depression received the treatment. The intervention consisted of 16 2-hour group sessions occurring over an 8-week period. The first 1-hour of each meeting was taken directly from the CWD-A manual. The second hour was based on the LIVE manual. The adolescents who completed the treatment $(n=8)$ improved from pretreatment to posttreatment and those who discontinued treatment $(n=2)$ did not. Improvements were maintained at 3 months follow-up. The treatment produced strong group cohesion and both adolescents and their guardians rated the intervention positively. These results provide initial evidence of the efficacy of complementing CWD-A with LIVE.
\end{abstract}

Keywords: Adolescent depression, cognitive-behavioral therapy, functional analytic psychotherapy.

\section{Introduction}

Cognitive behavior therapy (CBT) is the most widely studied psychosocial approach to treating depressive symptoms in adolescents and appears clearly superior to no treatment and possibly several other forms of intervention (Brent, Gaynor, \& Weersing, in press). Nonetheless, a substantial number of individual participants continue to meet criteria for a depressive disorder following acute CBT, suggesting the need for improvements. This paper offers a theoretical rationale for augmenting one form of CBT (the Adolescent Coping with Depression course: CWD-A); describes, based on this rationale, a treatment developed to be offered in conjunction with CWD-A; and explores the feasibility of combining CWD-A with this new intervention (called Learning through In-Vivo Experience: LIVE).

Reprint requests to Scott T. Gaynor, Western Michigan University, 3530 Wood Hall, Kalamazoo, MI 49008, USA. E-mail: scott.gaynor@wmich.edu 
The efficacy of individual cognitive-behavior therapy (CBT) for depressed adolescents has been established in three clinical outcome studies to date. These studies suggest that CBT outperforms no treatment, family therapy, supportive therapy, and relaxation training (Brent et al., 1997; Rosselló \& Bernal, 1999; Wood, Harrington, \& Moore, 1996), while producing results equal to interpersonal psychotherapy (Rosselló \& Bernal, 1999). It also appears that CBT for depressed adolescents applied in a brief, intensive, group format produces results superior to no treatment (Clarke, Rohde, Lewinsohn, Hops, \& Seeley, 1999; Kahn, Kehle, Jenson, \& Clark, 1990; Lewinsohn, Clarke, Hops, \& Andrews, 1990; Reynolds \& Coats, 1986). However, the data comparing group CBT to other treatments have yet to conclusively favor CBT over relaxation training, self-modeling, or a therapeutic support group (Fine, Forth, Gilbert, \& Haley, 1991; Kahn et at., 1990; Reynolds \& Coats, 1986).

Unfortunately, even when treatment appears efficacious at the group-level, there appears to be a substantial number of participants that continue to meet criteria for a depressive disorder following treatment. Combining the data from several studies using posttreatment diagnostic interviews suggests that $20-50 \%$ of participants continue to meet criteria for a depressive disorder after CBT (Brent et al., 1997; Jayson, Wood, Kroll, Fraser, \& Harrington, 1998; Lewinsohn, Clarke, Rohde, Hops, \& Seeley, 1996; Wood et al., 1996). Furthermore, a substantial number of adolescents receive additional treatment following acute CBT. For instance, Brent and colleagues found that $49 \%$ of the participants that received CBT in their clinical trial sought open treatment during follow-up (Brent, Kolko, Birmaher, Baugher, \& Bridge, 1999). Likewise, Clarke et al. (1999) report 29\% of adolescents received additional treatment during follow-up from CWD-A. These data suggest that, despite the efficacy established at the group-level, acute CBT was not sufficient for an important subset of participants.

Examination of the characteristics of those who failed to respond to acute CBT suggests non-responders are older, more severely impaired psychosocially, more severely depressed, higher in intake anxiety, engaging in fewer pleasant activities, higher in depressotypic cognitions, and more hopeless (Brent et al., 1997, Clarke et al., 1992; Jayson et al., 1998; Lewinsohn et al., 1996). Relatedly, pursuit of additional treatment after an initial acute intervention was predicted by greater severity of depressive symptoms, presence of comorbid disruptive behavior disorder, and familial difficulties in affective involvement (Brent et al., 1999). These data point to the need for improvements in acute CBT for depressed teens and sugest variables worthy of consideration in studies with this population.

One potential path to improving currently available interventions for adolescent depression is to augment treatments that already have some established validity. Such an approach allows one to build on existing data and to take advantage of established treatment protocols. For this study, we chose to augment the Adolescent Coping With Depression Course (CWD-A), which in two randomized controlled clinical trials has produced results superior to a waitlist condition (see Clarke et al., 1999; Lewinsohn et al., 1990). However, before pursuing the combination treatment in practice, there must be a theoretically driven rationale for the combined approach. Specifically, there must be reason to believe that the components of the treatments (i.e., CWD-A and LIVE) are not incompatible and that the additional treatment (i.e., LIVE) could provide something unique, that is not redundant with the original (i.e., CWD-A). The following sections provide a description of CWD-A and the theoretical rationale for the addition of LIVE. 


\section{CWD-A: Theory and practice}

CWD-A is based on a social learning theory of depression, which suggests that depressive symptoms are the outcome of a depressogenic cycle, which begins when adaptive behavior patterns are disrupted due to insufficient coping skills for effectively managing negative experiences and life events. The inability to skillfully manage "depression-evoking events" initiates, and then maintains, the depressogenic cycle (Clarke, Lewinsohn, \& Hops, 1990; Lewinsohn, Hoberman, Teri, \& Hautzinger, 1985).

Thus, CWD-A emphasizes skill acquisition to break or reverse the depressogenic process. Specifically, CWD-A focuses on teaching skills for increasing social reinforcement, pleasant events, problem-solving, and communication, while reducing depressive cognitions and anxiety (Clarke et al., 1990). The format of the CWD-A group is psychoeducational/didactic. That is, skills are initially taught by the group leader in a lecture format and are then practiced in role-play exercises, structured tasks, and group activities. Homework assignments are used to continue skill-building and increase generalization.

\section{Conceptual case for complementing CWD-A}

It is possible that CWD-A is quite successful in maximizing the amount of skill development that can be achieved through didactic presentations and structured practice, but that there is a limit on what can be accomplished with such an approach. If this is the case, other mechanisms through which skills can be learned need to be considered. One approach to specifying these other mechanisms comes from an understanding of the distinction made by behavior analysts between behavior that is largely verbally-governed (rule-governed behavior) versus that which is governed primarily by the prevailing environmental circumstances (contingency-governed). In these terms, CWD-A and other didactic therapies tend to emphasize rule-governed behavior (Hayes \& Ju, 1998; Hayes, Zettle, \& Rosenfarb, 1989). As an example, early in CWD-A, participants are taught skills in starting a conversation. Sample appropriate and inappropriate times for initiating conversations are discussed, as well as the type of questions most appropriate for use in beginning a conversation. In essence, a set of rules for conversation initiation are taught. To the extent that the rules accurately describe settings common in the natural environment, the chance for successful application increases (i.e., having a rewarding conversation). However, to the extent that these rules are insensitive to the environment conditions prevailing in a given situation, they are unlikely to produce a rewarding conversation even when explicitly followed. In the latter case, more subtle discriminations that go beyond what is specified in the rule are often required. For instance, another person may say "hello" because he/she is interested in conversing or because he/she is merely being polite. Initiating a conversation would likely produce quite different results across these two situations, despite the fact that both conform to the general guidelines of the rule.

In addition, assuming that the situation is one in which the specified response would be reinforced, the individual must adequately perform the behavior in that context. For instance, imagine an adolescent at a social gathering opening a conversation with " $\mathrm{Hi}$, how are you enjoying the party?"' versus "What are you doing here?" Both of these responses meet the criteria specified in the rule (i.e., ask open-ended questions), but the likelihood that the former will lead to a rewarding conversation appears much greater. 
The preceding examples point out how rules necessarily involve incomplete descriptions of the context and can only specify general, rather than specific, classes of behavior in which to engage. Of the utmost importance, however, is whether the rules lead to increased contact with important reinforcers. Notice, however, that during the CWD-A sessions, the reinforcers of greatest interest and likely impact (e.g., having a rewarding conversation with a peer) are not emphasized. The goal in the therapy session is to practice the general classes of behavior specified in the rule (e.g., generating open-ended questions) independent of their immediate in-session impact. Verbal praise from the therapist and group members about the teen's use of the skill being practiced serves as a substitute. The hope is that the skillful behavior will transfer outside of therapy where the other (naturally occurring) reinforcers will be received.

The preceding discussion is not designed to denigrate CWD-A or other didactic approaches, which have clearly established efficacy in controlled trials. Indeed, one might argue, the examples above are the exception rather than the rule. However, these examples point to potential reasons why skills taught via instruction and structured practice may not be maximally effective. First, because in natural environments (e.g., when not role-playing) these skills need to be flexibly applied depending on the situation and in the face of the individual's affect in the moment and, second, because the reinforcers of greatest interest are not received during the practice. In other words, there is a problem with generalization, both in terms of the client's behavior transferring smoothly to other situations, as well as the therapeutic environment being an effective proxy for the environment of interest outside therapy.

Behavior therapists often deal with such difficulties by taking the treatment directly into the environment of interest (e.g., doing a home visit with an OCD client). However, it is also possible that features of the environment of interest are often regularly present in the therapy setting, but are not recognized or maximally incorporated into the intervention (Kohlenberg \& Tsai, 1991). For instance, a great deal of problematic behavior occurs in interpersonal relationships and, thus, it might be anticipated that behavior that is problematic (or beneficial) for the client should be evoked in the therapy relationship. By focusing on client presenting problems that are evoked in the therapeutic relationship, consequences (i.e., punishment, extinction, reinforcement) can be applied immediately following client problem behaviors or improvements as they happen (Kohlenberg \& Tsai, 1991).

Based on this premise, Kohlenberg and Tsai (1991) have developed a therapeutic approach known as functional analytic psychotherapy (FAP). In FAP, Kohlenberg and Tsai (1991) distinguish three classes of client behavior (referred to as clinically relevant behavior or $\mathrm{CRB}$ ) occurring within the therapeutic milieu for which the therapist pays special attention. Briefly, a CRB1 is an instance of client problematic behavior occurring within the therapy session, while a CRB2 denotes client improvements witnessed in the session. CRB3's are instances where the client gives an interpretation of the contingencies controlling his/her behavior. Importantly, CRBs are idiographic and functional in nature, such that a CRB1 for one client may in fact be a CRB2 for another client. For maximum therapeutic benefit, the therapist must be vigilant for instances of $\mathrm{CRB}$, evoke $\mathrm{CRB}$, and reinforce CRB2s (Kohlenberg \& Tsai, 1991). The general idea is that once CRB is evoked, the opportunity for more powerful and naturalistic learning can occur in the here-and-now of the therapy setting. Moreover, it is hypothesized that because this learning occurs naturally, and not in a context of practice, the likelihood that behavior will generalize increases. 
Theoretically, the greater the similarity between the therapeutic environment and the client's everyday milieu, the greater the likelihood that relevant problematic social behavior will be evoked in session, thus increasing the potential opportunities for instructive feedback and client improvements. Therefore, group therapy, in which not only the therapist but also a small group of peers are present, may be the modality of choice (Budman, Bennett, \& Wisneski, 1981; Riester, 1994; Yalom, 1995). The presence of peers increases the likelihood that the therapy setting will share stimulus properties with the clients' everyday interpersonal relationships (e.g., the group as a "social laboratory"), has built-in components that are likely present across many interpersonal settings involving more than dyadic relating (e.g., increased social anxiety, developing first impressions, sharing time, etc.), and provides the opportunity for instructive peer feedback as opposed to simply therapist feedback.

In addition to the theoretical reasons offered above, existing empirical data and clinical impressions provide some rationale for suggesting that a focus on in-session interpersonal behavior, carried out in a group format, might be especially useful for depressed adolescents. Empirically, depression in adolescence is associated with numerous interpersonal impairments, including difficulties in familial relationships, poor peer relationships, having fewer social resources, less social competence, and increased social inhibition (Armsden, McCauley, Greenberg, Burke, \& Mitchell, 1988; Lewinsohn, Gotlib, \& Seeley, 1997; Puig-Antich et al., 1993). In addition, findings from clinical samples suggest that depressed adolescents are less successful in peer relationships than psychiatric controls (McCauley et al., 1993). Clinically, Brent et al. (1997) note that in their adaptation of Beck, Rush, Shaw and Emory's (1979) CBT for adolescents they put a greater emphasis on "cognitive distortions and affective shifts that occur during therapy sessions" (p. 878). Moreover, recognition is made in the CWD-A manual of the important socializing that occurs during informal discussions between the teens at the break in the meeting. In fact, some preliminary data suggest that adolescents in a process oriented support group tended to be more engaged and less avoidant compared to those in a social skills group (Fine et al., 1991).

\section{Complementing CWD-A with Learning through In Vivo Experience (LIVE)}

Based on the above considerations LIVE was developed. A manual was created which guided the group leader in the task of establishing an in-session focus and in creating an atmosphere where positive behaviors could emerge and be reinforced, and problematic (depressotypic) social behavior evoked and instructive feedback received. LIVE has strong ties to FAP (Kohlenberg \& Tsai, 1991); however, an important influence was also the experiential approach of Yalom (1995).

LIVE was developed as a possible augmentation strategy to group CBT. However, before the question as to whether LIVE can enhance CWD-A can be answered it must be established that LIVE and CWD-A can be used in conjunction with one another. The present study addressed this question by offering a combination treatment. The first one-hour of each session was spent in the traditional CWD-A fashion. That is, CBT skills were taught, reviewed, and practiced. The format of the second hour of each session was designed to focus on interpersonal learning in the social microcosm of the group. In other words, while the LIVE and CWD-A components were certainly not antithetical to each other, there was no attempt at full integration. 


\section{Method}

\section{Participants}

Ten adolescents, between the ages of 13 and 18, participated. Table 1 presents general demographic information for each participant. Participants were recruited from the community and were required to meet DSM-IV criteria for a depressive disorder (as assessed via the Diagnostic Interview Schedule for Children, DISC) or a cutoff score $>10$ on the Hamilton Rating Scale for Depression - Self-Report (HRSD) or $>13$ on the Beck Depression Inventory (BDI). In addition, eligible participants could not be actively suicidal; substance dependent or primarily in need of substance abuse treatment; currently receiving psychotherapy; or diagnosed with psychotic disorder, psychotic depression, bipolar disorder, cognitive delay, or organic brain syndrome. Adolescents who were currently taking antidepressant medication were admitted into the study if they had been on the same medication and dose for 2 months or more and met entry criteria. The adolescents were recruited in two cohorts, resulting in 2 groups (referred to as Group A and Group B), each consisting of 5 individuals. Despite both groups displaying significant symptoms of depression, the participants in Group B had a more severe current and historical clinical profile (see Table 2).

Following completion of two initial assessment meetings where inclusion criteria were assessed and baseline data collected, eligible participants were scheduled for a preparation meeting the week prior to the start of their group. During the preparation meeting both the adolescent and a guardian received psychoeducation about depression, including a social learning interpretation of depression and discussion of how this related specifically to the teen's symptomatic presentation.

\section{Experimental design}

The design was an open clinical trial format to assess the feasibility of the combined treatment and to collect initial outcome data. All participants received CWD-A+LIVE and no

Table 1. Demographic information

\begin{tabular}{lccccc}
\hline Participant & Age & Grade & Sex & $\begin{array}{c}\text { Living with } \\
\text { 2 parents }\end{array}$ & $\begin{array}{c}\text { Treatment } \\
\text { Protocol }\end{array}$ \\
\hline $\begin{array}{l}\text { Group A } \\
21\end{array}$ & 14 & 8 & F & Yes & Complete \\
22 & 18 & 12 & F & Yes & Complete \\
23 & 17 & 11 & M & Yes & Complete \\
24 & 15 & 10 & M & Yes & Complete \\
25 & 14 & 8 & F & Yes & Complete \\
& & & & & \\
Group B & 18 & 12 & F & No & Complete \\
05 & 16 & 9 & F & No & Complete \\
07 & 16 & 10 & M & No & Complete \\
08 & 14 & 7 & F & No & Incomplete \\
10 & 18 & 12 & F & No & Incomplete \\
11 & & & & & \\
\hline
\end{tabular}




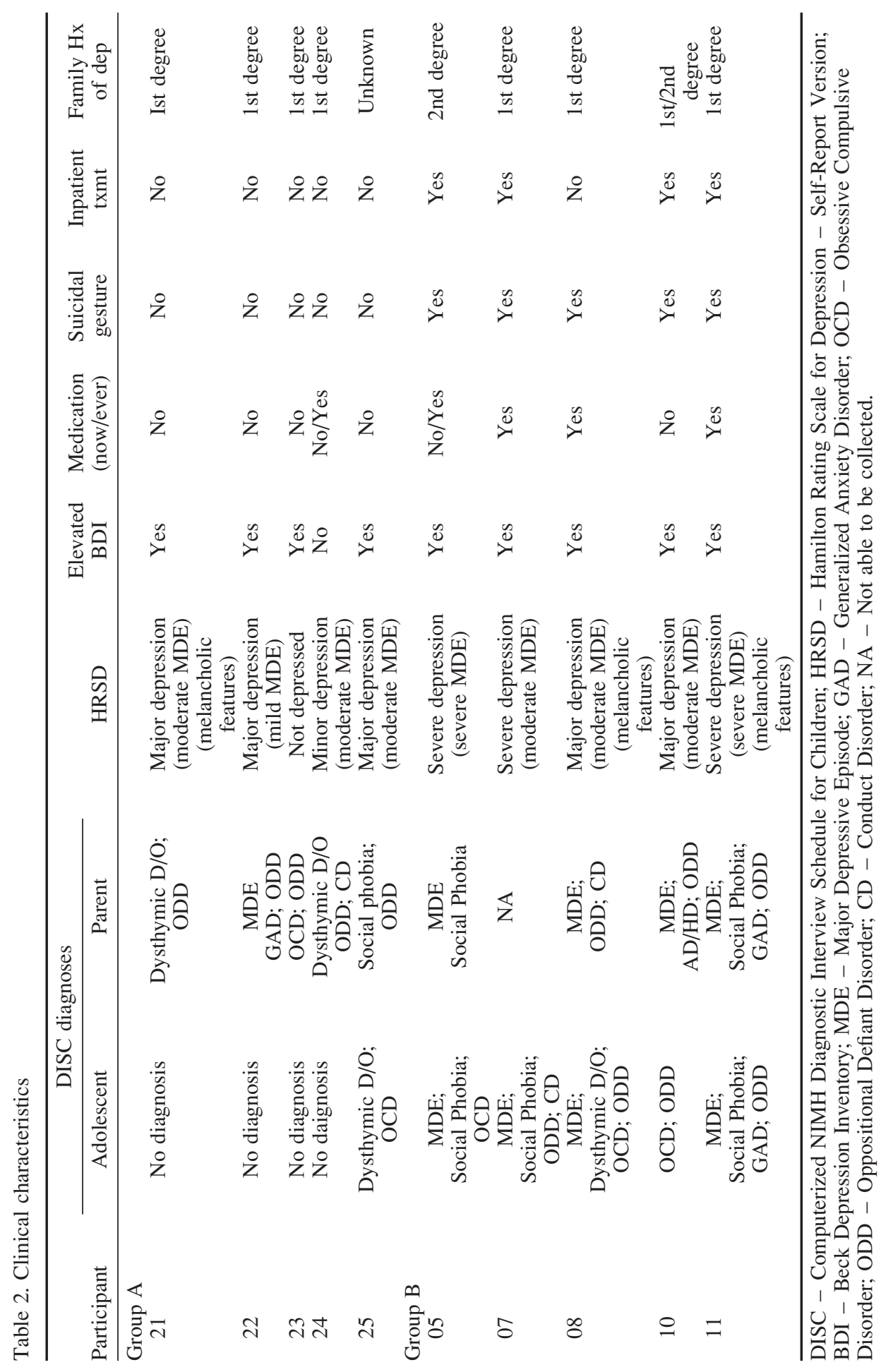


concurrent waitlist or attention-control group was utilized. Assessment measures were collected prior to treatment, throughout the course of treatment, at one-week following treatment completion, and at a 3 month follow-up period. The use of an open clinical trial format is consistent with the model adopted by the U.S. National Institutes of Health for the development of psychotherapeutic treatments (Najavits, 1998). The model suggests that developing therapies should progress through a series of 3 stages, with control conditions becoming successively more rigorous at each stage. The current study is consistent with the goals of stage 1: "develop the treatment and pilot test it with a basic design" (Najavits, 1998, p. 177). There was a heavy reliance on single-subject methods and a within-subject approach to assessing clinically significant change.

\section{Treatment}

The intervention consisted of 16 meetings delivered over an 8 week period, with each meeting lasting 2 hours. The content of the first 1-hour of each meeting was taken directly from the CWD-A manual. Because only 1-hour of each meeting was devoted to CWD-A, the entire treatment package could not be administered. The streamlined CWD-A included topic areas related to social learning theory of depression, friendliness skills, self monitoring, pleasant event scheduling, cognitive restructuring, and problem-solving. The order in which topics were presented was maintained as much as possible. However, some alterations were needed to ensure that enough time was available to completely present and practice each skill. The recommended homework practice from each skill was assigned. The second hour of each meeting involved the implementation of LIVE.

The practice of LIVE. As part of the broader introduction to the treatment, the adolescent and therapist collaborated to develop initial individualized goals for the LIVE portion of group prior to the first session. In collaborating with the adolescent to develop these individualized goals (referred to as In Group Goals for Yourself - IGGYs), the leader referenced material collected during the pretreatment assessment. Especially helpful was the Reasons for Depression Questionnaire (RFD; Addis, Truax, \& Jacobson, 1995), a self-report inventory where respondents rank statements as to the extent they believe them to be a cause of their depression. Relevant RFD items that were used in goal selection included: no one really understands me, I can't make friends, I'm not good at expressing my thoughts and feelings, or other people don't like me.

The first session of LIVE focused on giving the adolescents a framework for understanding the importance of, and how to engage in, interpersonal process work. Specifically, the adolescents were provided with a TV metaphor - actors versus directors - to focus them on the behaviors that they are asked to perform. They were told that each has two ongoing jobs in the group. The first job, that of being an actor, simply required that they be honest and be themselves. The second job, that of being a director, required that they provide positive and constructive feedback to the others (actors) to help them be as interpersonally effective as possible. After introducing the actor/director distinction the leader might say something such as:

In this group each of us is going to have two jobs to do at all times. First, we are our own actors and our job is to just be honest and to be ourselves. One of the best ways to do this is to make personal statements about how we are reacting to what is happening in the group 
and how it is making us feel right at that moment. Second, we will be directors, and as directors our job is to provide constructive feedback, support, and encouragement to other actors. Remember no sarcasm or meanness, but helpful coaching.

The remainder of the first session was focused on teaching the group members how to select goals appropriate for in vivo work (i.e., IGGYs). They were given two rules for in-group goals: 1) they must be something that can be observed in the group; 2) they must involve getting along with other people. Following the discussion of these requirements the group then worked with the leader to evaluate the appropriateness of several hypothetical in-group goals, sharpening their ability to identify when the requirements were met versus when modifications were needed.

During the second session each group member was, for the first time, required to state an IGGY that he/she would work towards that day. The purpose of having each teen state his/ her IGGY was to focus the teen on what he/she was to accomplish in the meeting and to facilitate the other members' ability to identify behavior for which feedback should be provided. In Kohlenberg and Tsai's (1991) terms, work towards an IGGY is a CRB2 worthy of reinforcement, while behavior opposite an IGGY is a CRB1 calling for corrective feedback or the blocking of avoidance. Some examples of IGGYs were the following: share more personal thoughts and feelings with the group, work on being a focused listener and ask direct questions of others to help them express thoughts and feelings, try to be more involved despite feeling shy, and express to the group when I am not feeling as involved as I would like.

Table 3 reviews the general structure of the LIVE sessions (2-16). At the beginning of each meeting the importance of an in-session focus was highlighted, the members' two jobs were reviewed, the parameters for choosing an IGGY were discussed, and each participant stated an IGGY for that meeting. This introductory period was followed by a discussion period, which, when possible, was strongly influenced by the adolescents' choice of material to discuss. However, when necessary, the leader utilized an activity to facilitate an in vivo focus. The goal of the activity was not to practice previously taught skills (as is usually the case in CBT), but to provide a context where CRB might emerge. For instance, in one meeting each member was asked to give him/herself a participation grade. The activity was not to practice skills in self-evaluation, but to encourage interpersonal process and to provide a context where clinically relevant behavior could emerge. For instance, if a normally selfcritical adolescent gave him/herself a good grade, this would be an improvement. However, if a self-critical teen gave him/herself a bad grade, others might offer disconfirming feedback. Following the discussion period, the last several minutes of each meeting were used to reflect on the group session, review progress on IGGYs, and conclude the meeting.

During the early sessions especially, the focus was on positive behavior and positive feedback, which was designed to increase self-esteem and help members capitalize on existing strengths. The leader was required to be very active in this process, modeling giving constructive feedback, asking for feedback, and receiving feedback openly. During both groups there was a notable decrease in reliance on the leader to guide and structure the interaction as the group progressed. This is not to say the leader was absent, but instead was able to spend less time providing structure and more time attending to the clinically relevant interaction patterns. Throughout, the leader actively attempted to facilitate the 1) identification of depressotypic interpersonal patterns, 2) delivery of positive feedback for improvements, and 3) recognition of how in-group depressotypic interpersonal patterns may 
Table 3. General overview of session structure for sessions 2-16. (Bolded quotes are statements made by the leader. Centered non-bolded text provides a summary of appropriate response content)

1. Review (10 minutes)

"What are the four guidelines for this class (Clarke et al., 1990)?"

Avoid depressive talk, share time, personal information should not be shared outside the group,and offer support/feedback

"What is the main purpose of the second hour of each meeting?"

Learn as much as possible about how we relate to others in the group.

"What are our two jobs that we have to be aware of at all times in group?",

Evoke responses - correct answers are 1) actor - be yourself and

2) director - provide constructive feedback to the actors

"What are the two requirements for an IGGY?"

Evoke responses - correct answer is 1) happens in the group

2) involves getting along with others

2. State IGGYs (10 minutes)

"Okay, now let's have each of you tell us about an IGGY that you would like to work on today. Let's go around the group and each person can share his or her IGGY with the others - remember your goal has to be one that you can work on in this group right here. Everyone else, the directors, listen closely because you'll need to watch for behavior related to these IGGYs to happen in group. Will someone volunteer to start us off?',

3. Process/Discussion component (30 minutes)

After each participant had stated an IGGY the meeting was opened up for a more general discussion. When possible, the teens were allowed to introduce the topics discussed in this section. It was hypothesized that the more the teens generated the material the more age- and peer-relevant stimulus properties would arise that related to their depressive symptoms. The leader's job was to be alert to the developing process and to participate in identifying and giving feedback for IGGYs and other relevant behavior.

"What's on people's minds today? Let's open things up a little bit now, but keep in mind our IGGYs and our 2 jobs to do: 1) be ourselves and be honest, 2) provide constructive feedback to each other."

In addition, the leader at times would introduce activities designed to potentially stimulate relevant interpersonal behavior or in order to focus the teens on in group behavior patterns

4. Wrap Up/Reflection/Conclusion - review progress on IGGYs (10 minutes)

This is a time for the leader to encourage the group to review the behavior towards IGGYs that occurred during the meeting and to provide some final summary information on the teen's behavior during the session. 
be related to daily life situations. Towards this end, the leader provided the adolescents with written summaries of their progress at the midpoint and conclusion of the treatment. These written summaries were discussed in group to facilitate attention to, and recognition of, goal-relevant behavior and as an activity that might stimulate an in-session focus or occasion clinically relevant behavior.

As the group progressed, the members were encouraged to give and receive not only positive feedback but also constructive feedback designed to help each learn as much as possible about their styles of interpersonal relating and how this relates to their depression and in-session goals. Interestingly, the leader's impression from the CWD-A+LIVE open clinical trial was that the adolescents were much more comfortable and willing to provide each other with positive feedback. This balance toward the positive appeared especially appropriate given the lack of social supports and positive interpersonal contexts depressed teens often report. This is not to say that no constructive feedback was offered, however. In fact, the leader recognized that one of the strengths of the teenagers was in blocking avoidance. Because they were unlikely to be cast as authority figures, at times they could be much more direct with each other than could the leader. However, there were also instances where the teens would validate avoidance making alternative interpretations difficult to explore. There were several occasions, for instance, when one member would offer a trait hypothesis, suggesting to another teen that a potentially problematic response was "just the way you are."

Toward the end of both groups, the members commented that many would not likely have become friends under the natural social selection processes. Yet, all came to value each other's opinion, form a cohesive group together, and receive a great deal of positive reinforcement from each other. Moreover, by the end of the group several members reported on how they would have misjudged others in the group based on initial impressions. These impressions suggested that the age-relevant stimulus properties (largely physical and appearance-based) were apparent and impacted initial impressions, but were largely modified or supplanted by impressions based on in-group interactions. As termination approached, the leader was again required to be more active, directing the group towards exploration of what they had learned about themselves in the relationships developed and the impact of these relationships ending.

Treatment integrity. The therapist (STG) completed a 16-item measure of treatment compliance immediately following each session. The first 10 questions consisted of the measure developed by Lewinsohn, Clarke and colleagues (Clarke, 1998; Clarke et al., 1995, 1999; Lewinsohn et al., 1990) and assessed adherence to the CWD-A manual. The last 6 items were added to address compliance with the goals of LIVE. The questions assessed the presence of particular components of the therapy and were rated on a 3 -point scale $(0=$ no, 1 = partial, 2 =yes). A doctoral level psychologist, experienced in CBT, FAP and conducting skill-based and process-oriented therapy groups, rated $25 \%$ of the sessions to provide reliability data. The sessions were chosen in a quasi-random fashion, with one tape randomly selected from sessions 1-4, 5-8, 9-12, and 13-16. For Group A sessions 1, 6, 10, and 16 were rated, while for Group B sessions 3, 8, 9, and 15 were rated. Interrater reliability according to the coefficient Kappa was .61 $(\mathrm{SE}=.10)$, which is consistent with the average Kappa of .69 reported by Lewinsohn et al. (1990) and suggests good agreement between raters. 
The adherence totals for the CWD-A portion and the LIVE portion were tallied separately, divided by the possible perfect score, and multiplied by 100 to produce a percentage adherence rating. The results are indicative of high adherence to the treatment protocols. For the CWD-A portion, and adherence ratings of the therapist and independent rater were $95 \%$ and $90 \%$ respectively. These results are in line with the average adherence rates of $86-94 \%$ previously reported in CWD-A clinical trials (Clarke et al., 1995, 1999; Lewinsohn et al., 1990). The ratings for the LIVE component, while suggesting protocol adherence, were not unexpectedly lower ( $80 \%$ and $82 \%$ respectively) according to both the therapist and the independent rater.

\section{Measures}

Diagnostic interview. The computerized NIMH Diagnostic Interview Schedule for Children (DISC), a clinician administered structured interview, was used to assess psychopathology across the major diagnostic categories of the DSM-IV at pretreatment and posttreatment. The modules assessing social phobia, generalized anxiety disorder, obsessive-compulsive disorder, major depression and dysthymic disorder, bipolar disorder, oppositional defiant disorder, and conduct disorder were administered to the adolescent (DISC-a) and, when possible, a parent (DISC-p). In addition, the items from the depression module assessing symptoms occurring during the 4 weeks prior to the interview were recorded separately allowing for calculation of the proportion of items endorsed. The DISC is considered a reliable tool with moderate to good validity in assessing child psychopathology (Schwad-Stone et al., 1996; Shaffer et al., 1996).

Depression. Participants completed the 76-item self-report version of the Hamilton Rating Scale for Depression (HRSD), which is based on the frequently used semi-structured interview, at pretreatment, posttreatment, and 3-month followup. The 21-item Beck Depression Inventory (BDI; Beck, Ward, Mendelson, Mock, \& Erbaugh, 1961; Roberts, Lewinsohn, \& Seeley, 1991) was administered weekly from pre to posttreatment and at 3-month followup.

Treatment participation, satisfaction, and experience. The Yalom Group Cohesiveness Scale (YGCS; Yalom, 1995) is a 10-item self-report inventory, scored on a 1-5 Likert scale, measuring feelings of belonging, acceptance, and approval among group members. The scale was slightly modified to facilitate completion by adolescents. Higher scores suggest greater cohesion. The 8-item Client Satisfaction Questionnaire (CSQ; Larsen, Attkisson, Hargreaves, \& Nguyen, 1979), scored on a 1-4 Likert scale, measured client satisfaction with treatment. Higher scores suggest greater satisfaction. The CSQ was administered to both the teen and a guardian at posttreatment. The 10-item Treatment Experiences Questionnaire (TEQ), scored on a 1-5 Likert scale, was developed by the authors to assess participants' experience across the two components of the group. The TEQ includes questions related to the differential utility and distinguishability of CWD-A and LIVE (e.g., During which hour of the group did you learn the most about . . .). Lower item scores (i.e., 1 or 2) represent the CWD-A component and higher scores (i.e., 4 or 5) represent the LIVE component. 


\section{Results}

\section{Depression}

The weekly BDI data for Group A are presented in Figure 1. The horizontal lines mark the BDI cutoff for return to normative functioning and the asterisks denote the point at which the reliable change index (RCI; Jacobson \& Truax, 1991), if reached, was maintained below the .05 level. Examination of Figure 1 reveals a decrease in BDI scores for each participant beginning early in the course of treatment. Specificlly, there was a small but consistent decrease from baseline at week 1 for participants 21, 22, 24, and 25, while P23 reported complete symptom remission after 1 session. Unlike P23, the other 4 participants showed more progressive reductions in their BDI scores; however, the majority of change was experienced in the early weeks of treatment. For instance, P24 had a very low BDI score by the second week of treatment (after 3 sessions) and P21 met the BDI cutoff in week 2 (after 3 meetings) and satisfied the RCI in week 3 (after 5 sessions). In addition, both P22 and P25 met the BDI cutoff in week 3 (after 5 sessions), while sustained statistical significance on the RCI occurred at week 5 (9 sessions into treatment). These data suggest that by approximately the mid-point all participants demonstrated significant change, which was maintained through the remainder of the treatment and at 3-month follow-up. Figure 2 shows the pretreatment, posttreatment and follow-up data on the HRSD, DISC-a, and DISC-p, which confirm the BDI results. Thus, with the exception of P23, the results are quite consistent across measures.

Weekly BDI data for Group B are presented in Figure 3. Data from participants 05, 07, 08, and 10 mirror that observed with Group A in that there was an initial decrease in BDI score after 1 session. This initial decrease was not observed for P11. However, unlike Group A, only P07 went on to demonstrate a marked decrease in BDI score over the course of treatment. As Figure 3 demonstrates, P07 met the BDI cutoff criteria in week 2 (after 3 sessions) and achieved sustained clinical significance on the RCI at week 5-6 (9-11 sessions into treatment), which was maintained through the 3-month follow-up. P05 and P08 showed a decrease in depressive symptoms compared to their pretreatment baselines; however, both failed to sustain scores below the cutoff and neither met the RCI criterion. The results of the HRSD, DISC-a, and DISC-p (see Figure 2) confirm that P07 showed the most sizable change, while P05 and P08 showed symptomatic improvement, but failed to achieve remission.

Those who discontinued the treatment prior to completion (P10 \& P11) showed no functional change in depressive symptoms from pretreatment to posttreatment. As Figure 3 shows, P10 had an initial drop in BDI score; however, this was not sustained the following week nor at the posttreatment assessment. Likewise no positive changes were observed according to the HRSD (see Figure2). P11 showed a decreasing trend in BDI scores while attending treatment; however, this improvement never met the BDI cutoff or RCI criterion. Moreover, at posttreatment P11 reported an increase in symptoms on the DISC-a, and remained in the Severe Depression range on the HRSD (see Figure 2 and Table 3). These data suggest that unlike those who completed the treatment, those who discontinued treatment failed to show symptomatic improvement at posttreatment. The data are less clear at 3-month follow-up as P10 reported complete symptom remission, while P11 reported sustained symptoms. 

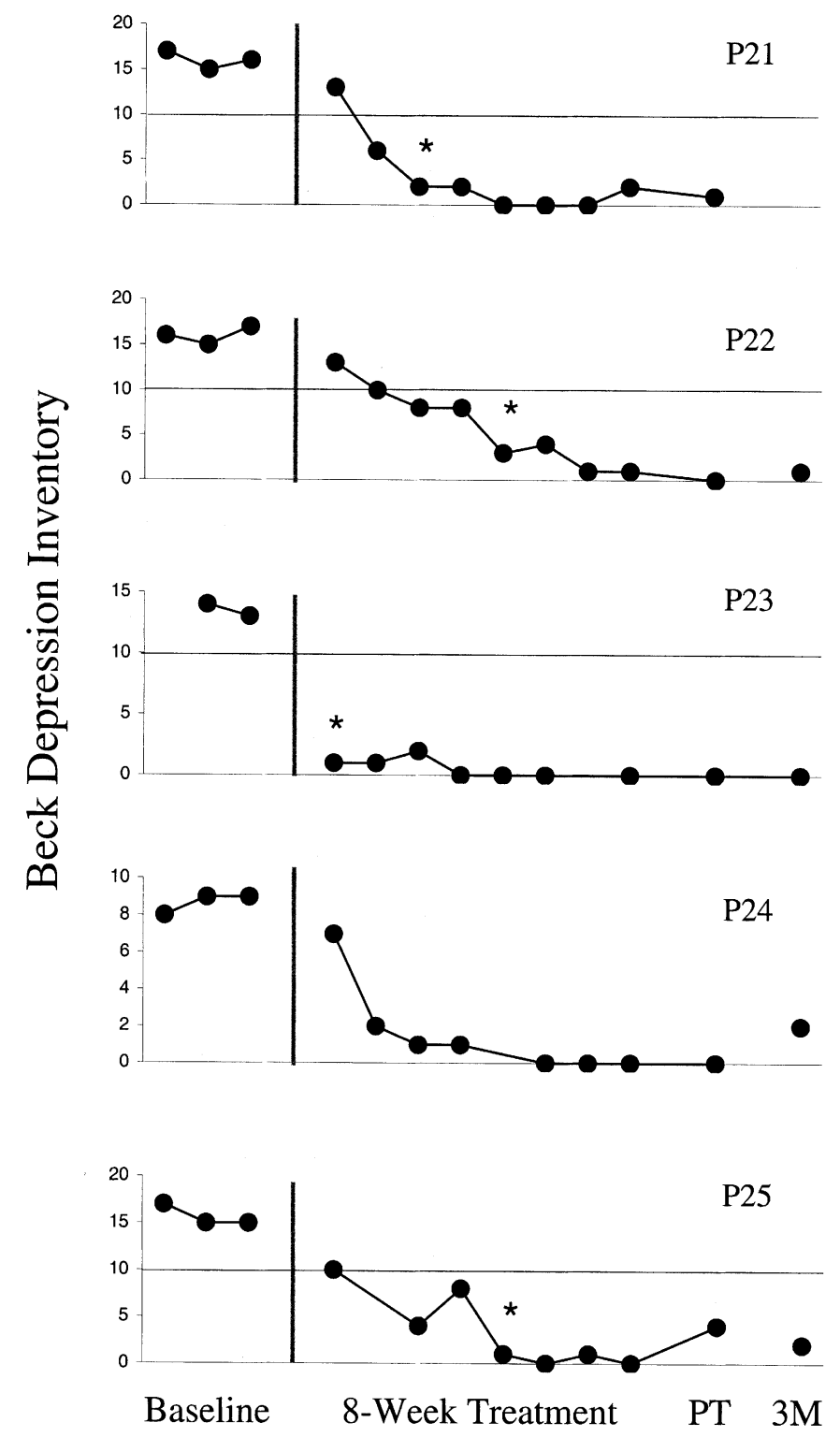

Figure 1. Time-series plots of BDI scores for each participant in Group A. The vertical line marks the BDI cutoff and asterisks mark the point at which the RCI criterion was met and maintained. PT Posttreatment; $3 \mathrm{M}-3$-month follow-up. Note: This study used a cutoff of $\leq 9$ on the BDI (similar to Elkin et al., 1989) to mark return of functioning within the normative range. When the cutoff was reached and the reliable change index (RCI) was statistically significant at the .05 level, the result was considered clinically significant (Jacobson and Truax, 1991). Clinically significant change could not be calculated for P24 given his low initial BDI; however, there was evidence of return to normative functioning based on the change in HRSD score from the Minor Depression to Nondepressed range. In addition, based on the BDI profile it was guessed that most of the change occurred within 3 sessions. 
BL DPT $3 \mathrm{M}$
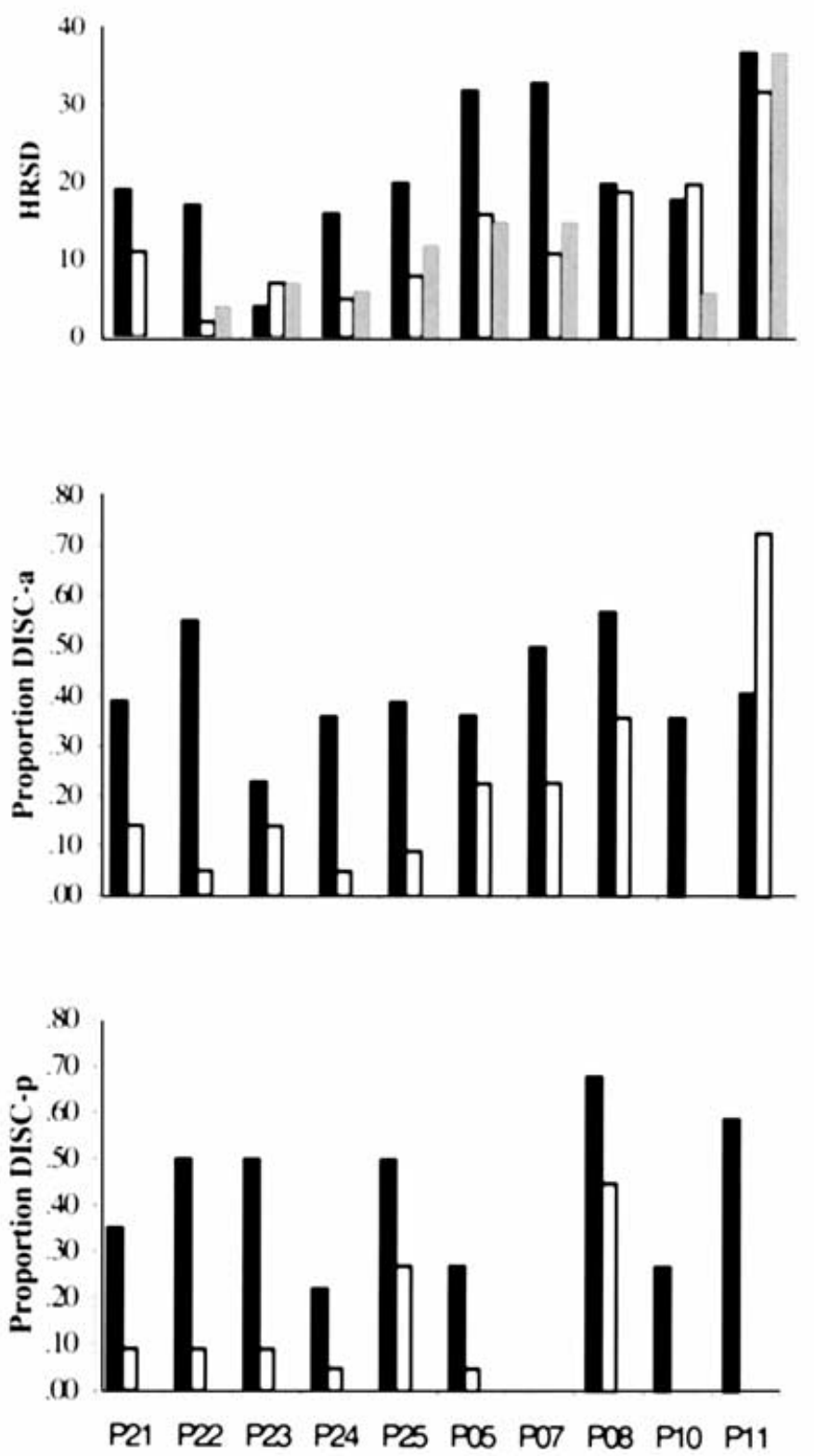

Figure 2. Pretreatment baseline (BL; filled bars), posttreatment (PT; open bars) and 3-month follow-up (3M; grey bars) data. DISC-a and DISC-p data are presented as proportion of items endorsed during the 4 weeks preceding the interview. DISC-a and DISC-p data were not collected at 3-month follow-up. HRSD scores between 0 and 10 are considered indicative of no depression, while scores ranging from 11-16 suggest mild depression, 17-25 indicate moderate depression, and 26+ severe depression. When bars are missing it is because data were unable to be collected. 

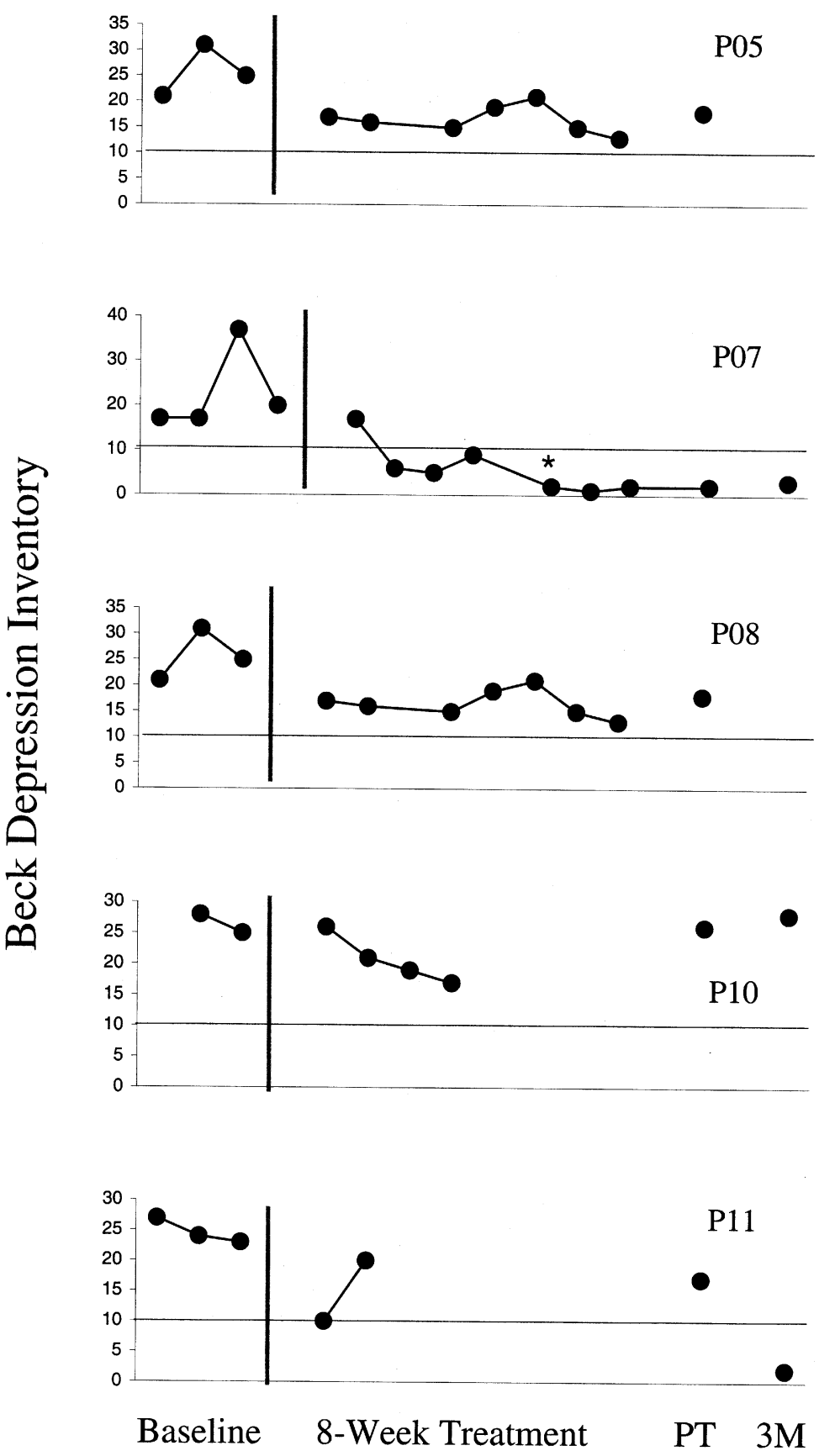

Figure 3. Time series plots of BDI scores for each participant in Group B. The vertical line marks the BDI cutoff and an asterisk marks the point at which the RCI criterion was met and maintained. PT - Posttreatment; 3M - 3-month follow-up. 


\section{Treatment participation, satisfaction, and experience}

Among treatment completers there was no difference in the number of sessions attended, as the means for both Group A $(M=14.10, S D=.74)$ and Group B $(M=13.50, S D=.50)$ were high. In addition, both groups reported high group cohesion at the end of treatment ( $M=50.0, S D=0.00$ and $M=42.0, S D=2.00$, for Group A and B respectively), which was exceptionally high for Group A. The mean scores according to the 4-point Likert scale of the CSQ were equally high for both participants in Group A $(M=3.83, S D=.16)$ and Group B $(M=3.50, S D=.20)$ and their primary caretakers $(M=3.78, S D=.12$ vs. $M=$ $3.69, S D=.27$, respectively).

The TEQ assessed whether, based on the participants' experience, the important foci associated with CWD-A or LIVE occurred during the expected portion of the session. Examination of the TEQ items indicate that participants clearly reported learning more about changing cognitions $(M=1.1, S D=0.35)$, applying problem solving skills $(M=1.8, S D=$ $0.71)$, and improving mood through behavioral activation $(M=1.6, S D=0.74)$ during the CWD-A component. In addition, they reported receiving greater interpersonal feedback $(M=$ $4.4, S D=0.52)$ and learning more about self-expression $(M=3.8, S D=1.16)$ and their interpersonal presentation $(M=3.8, S D=1.28)$ during the LIVE component. These data suggest that the participants experienced the treatment as it was designed and, therefore, provides additional evidence of treatment integrity. Also of interest from the TEQ, the CWD-A and LIVE components were rated as equally beneficial overall $(M=3.0, S D=$ 1.07). However, LIVE was rated as more enjoyable $(M=4.3, S D=0.71)$, while CWD-A tended to be rated more challenging $(M=2.4, S D=1.19)$ and informative $(M=2.5, S D=$ 1.07).

\section{Discussion}

\section{General discussion}

The results of this open clinical trial are consistent with the results of larger randomized controlled clinical trials in supporting the efficacy of a psychosocial intervention in reducing depressive symptoms among adolescents (Brent et al., 1997; Clarke et al., 1999; Lewinsohn et al., 1990; Rosselló \& Bernal, 1999). In particular, these results support the efficacy of CWD-A providing independent replication, albeit with a small sample, of the results reported by Lewinsohn, Clarke and colleagues. Furthermore, CWD-A was effectively streamlined and combined with LIVE, an approach emphasizing the application of consequences for interpersonally relevant behavior occurring within the treatment session. Furthermore, the development of LIVE drew heavily from a behavior analytically-based approach known as FAP (Kohlenberg \& Tsai, 1991), providing some of the first outcome data of its efficacy, ability to be flexibly applied in combination with other approaches, and portability to other research groups. Now that it appears clear that the two can be offered together, future research employing a dismantling design comparing CWD-A, LIVE, and CWD-A+LIVE can be considered to address questions related to the unique effects of the treatments and the added effect of the combination.

Pragmatically, it was suggested that CWD-A emphasizes psychoeducation and in-session structured practice to prepare the individual for the situations and consequences of interest, which occur outside the therapy session. LIVE, on the other hand, emphasizes immediate 
consequences for behavior evoked during the group, a context that is hypothesized to share functional similarities with settings the client experiences outside of therapy. The important differences are in the manner in which important behavior is generated, recognized, and reinforced, not in the particular techniques employed. As such, from a cognitive perspective LIVE might be viewed as an attempt to focus on in-session hypothesis testing using "hot" cognitions rather than "cold" cognitions (Greenberg \& Safran, 1984) or as an attempt to import behavioral experiments, designed to elucidate and confront pessimistic expectations and distorted thoughts through direct experience, to the therapy setting.

Conceptually, the differences between CWD-A and LIVE were described in terms of the relative emphasis on rule-governed behavior versus contingency-governed behavior. There was no suggestion, however, that LIVE completely eschews development of verbally-based rules for governing behavior (e.g., "when I have a negative thought use the A-B-C method") or that verbal rules can not come to merge with controlling variables in the environment. Similarly, it is not claimed that CWD-A completely precludes contact with immediate social consequences. Thus, from a conceptual or pragmatic position there is no reason a more complete integration of CBT/CWD-A and FAP/LIVE approaches could not occur. In fact, Kohlenberg and Tsai (1994) describe an integration of FAP and CBT referred to as FAP-enhanced cognitive therapy, where the emphasis is not only on using the therapeutic relationship, but also on identification and modification of in-session instances of cognitive distortion (see also Jacobson, 1994).

\section{Treatment outcome}

According to measures of clinical significance, only $1 / 3$ participants in the more severely depressed group reached remission compared to $5 / 5$ in the moderate group. Clarke et al. (1992) reported that both "Recovered"' and "Not Recovered" participants from the Lewinsohn et al. (1990) clinical trial demonstrated similar overall declines in BDI scores (14.6 and 13.7, respectively). What differentiated the groups was that the participants who came to be called "Recovered" began with lower depression scores, such that the change moved them into the range considered normative. This explanation accurately describes the current data, as the magnitude of BDI change was 13.3 and 13.4 for Group A and Group B (completers), respectively. A simple comparison of magnitude of BDI changes between Lewinsohn and colleagues (1990) and the present study does not suggest the addition of LIVE increased overall efficacy with respect to depressive symptom change. However, additional research assessing treatment effects across multiple outcome domains (e.g., cognitive, interpersonal, etc.) is needed to determine any incremental efficacy associated with the addition of LIVE.

It appears that more severely depressed adolescents with greater psychosocial impairments require additional or lengthier treatment to meet criteria for clinically significant change. The individual time-series data from those who failed to demonstrate clinically significant change (P05 and P08) do not reveal clear trends in the direction of decreasing symptoms as treatment progressed. This casts doubt on the efficacy of simply continuing the current treatment as a solution to the lack of complete remission, arguing potentially for an alternative treatment. For those adolescents who were moderately depressed, acute CWD-A+LIVE appeared sufficient to achieve remission. As noted above, 5/5 participants in Group B met criteria for clinically significant change. This is not an unimportant finding 
as all 5 of these participants, and their guardians, endorsed significant symptoms of depression at pretreatment. However, this finding does coalesce with other findings that responders to CBT tend to be less severely impaired than nonresponders (Clarke et al., 1992; Jayson et al., 1998).

An advantage to a methodology involving a small sample and repeated measures is that one can explore in greater detail the process of change. One interesting finding in the current sample is that $8 / 10$ participants showed at least a small, but visually noticeable, decrease in depressive symptoms at week 1 (measured prior to the second weekly session). During the first treatment session, social learning theory was presented and the participants were trained and assigned to self-record their mood. Initiation of self-monitoring often produces reactivity, a small change in the therapeutic direction (Baird \& Nelson-Gray, 1999). The initial small changes in depression for P05, P08, P21, P22, P24, and P25 are consistent with this effect. The large changes for P10 and P23 are unlikely attributable solely to reactivity; however, the impact of reactivity cannot be ruled out entirely. The initial change for P10 was transient, returning to approximately baseline level by the following week and remaining high at posttreatment. However, the large change for P23 was stable, remaining very low throughout treatment.

The large change following 1 treatment session may shed some doubt on the validity of the data for P23. In fact, only 2 pretreatment BDIs were collected from this participant and entry criteria were met solely on the BDI. Thus, it could be argued that P23's baseline did not represent stable and significant depressive symptoms. However, even when participants are required to meet diagnostic criteria, such a result is not completely unexpected. For example, Renaud et al. (1998) found that $31 \%$ of their sample showed a $50 \%$ or greater BDI decrease from pretreatment to session 2 and that such rapid responders tended to maintain these gains.

Among those who were functioning in the normative range at posttreatment, the majority of BDI change appeared to take place in $\leq 5$ weeks of treatment. Such rapid improvement is consistent with a review of CBT time course data with adults reporting that between 60 and $70 \%$ of change in depression occurred within the first 4 weeks of CBT (Ilardi \& Craighead, 1994).

\section{Level of satisfaction attrition}

As adolescents often enter treatment at the insistence of concerned adults, rather than through self-referral, it is important to find treatments in which they become invested and report satisfaction (Kazdin, 2000). CWD-A+LIVE appeared to fare well in this domain. Participants from both groups and their primary guardians reported a high level of satisfaction with the treatment. As standard CBT approaches with depressed adolescents are often completed in approximately 16 1-hour sessions (e.g., Brent et al., 1997) or less (e.g., Wood et al., 1996) the streamlined CWD-A should not be viewed as a diluted CBT. Thus, given the preliminary data that CWD-A+LIVE fared as well as standard CWD-A (in other studies), the greater enjoyment of the LIVE portion may be another potential reason to recommend its inclusion.

It is important to recognize that $2 / 10$ participants discontinued treatment with CWD-A+LIVE, suggesting that not every participant was completely satisfied. However, the $20 \%$ incompletion rate observed was commensurate with data from larger randomized 
clinical trials where incompletion rates of 14-27\% have been reported (Brent et al., 1997; Clarke et al., 1999; Lewinsohn et al., 1990; Rosselló \& Bernal, 1999). These preliminary data suggest that despite the greater emphasis on in-session interpersonal interactions, CWD-A+LIVE appears well-liked by the majority of participants and does not appear to confer any greater risk of treatment discontinuation compared to other approaches.

\section{Limitations}

As in any initial feasibility study, there were numerous limitations in the present design. First, the sample size was small, which has implications for the generalizability of the findings and highlights the importance of replicating these results with a larger sample and a more controlled design. Secondly, the absence of random assignment with a concurrent control group does not allow for the results to be conclusively attributed to the current treatment. It is questionable whether including a wait-list control condition could be ethically justified given previous studies demonstrating CWD-A to be superior to waitlist controls. However, future studies might consider using a dismantling strategy (described above) or comparisons with an attention control condition or another form of therapy (e.g., family therapy, supportive therapy), as CWD-A has only been tested against a waitlist control group. Due to the lack of an attention control condition, it is possible to attribute the current results to nonspecific factors such as having regular contact with the leader and a group of peers, rather than the proposed "active" ingredients of the treatment. A third liability of the present study is the combination CWD-A+LIVE design does not allow for ascertainment of the unique effects of LIVE. The decision to offer the combination treatment, as opposed to simply offering LIVE alone, was made for several reasons. First, the authors wanted to offer participants a treatment for which empirical support existed, while at the same time solidifying the LIVE approach. The combination treatment allowed for this possibility. In addition, LIVE was not conceptualized as a replacement for CWD-A, but rather as providing a component that was not emphasized in traditional CWD-A. It does appear the CWD-A and LIVE components were conducted and experienced uniquely, but the unique impact on functioning remains an open question.

Another significant limitation involves the use of only one therapist. The first author served as the leader for both groups; thus, the effects attributable to the therapist cannot be discriminated from those due to the therapy provided. As it was anticipated that the sample size would be small, use of multiple therapists would have served to simply introduce additional uncontrolled variance into the design. In addition, the first author conducted all pre and posttreatment assessments. Thus, it is not possible to rule out the possibility that the adolescents responded in a manner they hoped would please the investigator (although this is norm when measures are administered in clinical practice). To provide some protection from demand characteristics, all self-report inventories administered included a cover letter reminding participants not to place their name on the material (all items were pre-coded) and encouraging them to be honest in providing the information. With respect to the diagnostic interviewing, the DISC interview was chosen, in part, because respondents answer "yes" or "no", which eliminated the need for clinical judgment. Regardless, future research will need to demonstrate that other skilled therapists can implement LIVE and that the effects obtained herein hold when blind raters conduct the pre and posttreatment assessments. 


\section{Acknowledgements}

This article is based on the dissertation of the first author, in partial fulfillment of the requirements for the doctoral degree. The authors thank Dr Susan P. Keane, Director of the University of North Carolina at Greensboro Psychology Clinic, for use of space and resources necessary for conducting this study. The authors also thank Dr Rosemery O. Nelson-Gray and Dr Susan C. Baird for their input and thoughtful comments on an earlier version of this manuscript.

\section{References}

Addis, M. E., Truax, P., \& Jacobson, N. S. (1995). Why do people think they are depressed? The reasons for depression questionnaire. Psychotherapy, 32, 476-483.

Armsden, G. C., McCauley, E., Greenberg, M. T., Burke, P. M., \& Mitchell, J. R. (1990). Parent and peer attachment in early adolescent depression. Journal of Abnormal Child Psychology, $18,683-697$.

Baird, S. C., \& Nelson-Gray, R. O. (1999). Direct observation and self-monitoring. In S. C. Hayes, D. H. Barlow \& R. O. Nelson-Gray, The scientist practitioner: Research and accountability in the age of managed care (2nd ed., pp. 353-386). Boston: Allyn and Bacon.

Beck, A. T., Rush, A. J., Shaw, B. F., \& Emory, G. (1979). Cognitive therapy of depression, New York: Guilford Press.

Beck, A. T., Ward, C. M., Mendelson, M., Mock, J. E., \& Erbaugh, J. K. (1961). An inventory for measuring depression. Archives of General Psychiatry, 4, 561-571.

Brent, D. A., Gaynor, S. T., \& Weersing, V. R. (in press). Cognitive behavioral approaches to the treatment of depression and anxiety. In M. Rutter and E. Taylor (Eds.), Child and adolescent psychiatry: Modern approaches (4th ed.) Oxford: Blackwell Scientific Publications.

Brent, D. A., Holder, D., Kolko, D., Birmaher, B., Baugher, M., Roth, C., Iyengar, S., \& Johnson, B. A. (1997). A clinical psychotherapy trial for adolescent depression comparing cognitive, family, and supportive therapy. Archives of General Psychiatry, 54, 877-885.

Brent, D. A., Kolko, D., Birmaher, B., Baugher, M., \& Bridge, J. (1999). A clinical trial for adolescent depression: Predictors of additional treatment in the acute and follow-up phrases of the trial. Journal of the American Academy of Child and Adolescent Psychiatry, 38, 263-270.

Budman, S. H., Bennett, M. J., \& Wisneski, M. J. (1981). An adult developmental model of short-term group psychotherapy. In S. H. Budman (Ed.), Forms of brief therapy, New York: Guilford Press.

Clarke, G. N. (1998). Intervention fidelity in the psychosocial prevention treatment of adolescent depression. Journal of Prevention and Intervention in the Community, 17, 19-33.

Clarke, G. N., Hawkins, W., Murphy, M., Sheeber, L. B., Lewinsohn, P. M., \& Seeley, J. R. (1995). Targeted prevention of unipolar depressive disorder in an at-risk sample of high school adolescents: A randomized trial of a group cognitive intervention. Journal of the American Academy of Child and Adolescent Psychiatry, 34, 312-321.

Clarke, G. N., Hops, H., Lewinsohn, P. M., Andrews, J., Seeley, J. R., \& Williams, J. (1992). Cognitive-behavioral group treatment of adolescent depression: Prediction of outcome. Behavior Therapy, 23, 341-354.

Clarke, G. N., Lewinsohn, P. M., \& Hops, H. (1990). Instructor's manual for the adolescent coping with depression course. Eugene, OR: Castalia Press.

Clarke, G. N., Rohde, P., Lewinsohn, P. M., Hops, H., \& Seeley, J. R. (1999). Cognitivebehavioral treatment of adolescent depression: Efficacy of acute group treatment and booster sessions. Journal of the American Academy of Child and Adolescent Psychiatry, 38, 272-279. 
Elkin, I., Shea, M. T., Watkins, J. T., Imber, S. D., Sotsky, S. N., Collins, J. F., Glass, D. R., Pilkonis, P. A., Leber, W. R., Docherty, J. P., Fiester, S. J., \& Parloff, M. B. (1989). National Institute of Mental Health Treatment of Depression Collaborative Research Program: General effectiveness of treatments. Archives of General Psychiatry, 46, 971-982.

Fine, S., Forth, A., Gilbert, M., \& Haley, G. (1991). Group therapy for adolescent depressive disorder: A comparison of social skills and therapeutic support. Journal of the American Academy of Child and Adolescent Psychiatry, 30, 79-85.

GreenberG, L. S., \& Safran, J. D. (1984). Integrating affect and cognition: A perspective on the process of therapeutic change. Cognitive Therapy and Research, 8, 559-578.

Hayes, S. C., \& Ju, W. (1998). Rule-governed behavior. In W. O’Donohue (Ed.), Learning and behavior therapy. Boston: Allyn and Bacon.

Hayes, S. C., Zettle, R. D., \& Rosenfarb, I. (1989). Rule-following. In S. C. Hayes (Ed.), Rulegoverned behavior: Cognition, contingencies, and instructional control. New York: Plenum.

Ilardi, S. S., \& Craighead, W. E. (i994). The role of nonspecific factors in cognitiveBehavior therapy For DePRession. Clinical Psychology: Science and Practice, 1, 138-156.

JACOBSON, N. S. (1994). Behavior therapy and psychotherapy integration. Journal of Psychotherapy Integration, 4, 105-119.

Jacobson, N. S., \& Truax, P. (1991). Clinical significance: A statistical approach to defining meaningful change in psychotherapy research. Journal of Consulting and Clinical Psychology, 59, 12-19.

Jayson, D., Wood, A., Kroll, L., Fraser, J., \& Harrington, R. (1998). Which depressed patients respond to cognitive-behavioral treatment? Journal of the American Academy of Child and Adolescent Psychiatry, 37, 35-39.

Kahn, J. S., Kehle, T. J., Jenson, W. R., \& Clarke, E. (1990). Comparison of cognitive-behavioral, relaxation, and self-modeling interventions for depression among middle-school student. School Psychology Review, 19, 196-211.

KaZdin, A. E. (2000). Developing a research agenda for child and adolescent psychotherapy. Archives of General Psychiatry, 57, 829-835.

Kohlenberg, R. J., \& Tsai, M. (1991). Functional analytic psychotherapy: Creating intense and curative therapeutic relationships. New York: Plenum Press.

Kohlenberg, R. J., \& TsaI, M. (1994). Improving cognitive therapy for depression with functional analytic psychotherapy: Theory and case study. Behavior Analyst, 17, 305-319.

Larsden, D. L., Attkisson, C. C., Hargreaves, W. A., \& Nguyen, T. D. (1979). Assessment of client/patient satisfaction: Development of a general scale.Evaluation and Program Planning, 2, 197-207.

Lewinsohn, P. M., Clarke, G. N., Hops, H., \& Andrews, J. (1990). Cognitive-behavioral treatment for depressed adolescents. Behavior Therapy, 21, 385-401.

Lewinsohn, P. M., Clarke, G. N., Rhode, P., Hops, H., \& Seeley, J. R. (1996). A course in coping: A cognitive-behavioral approach to the treatment of adolescent depression. In E. D. Hibbs \& P. S. Jensen (Eds.), Psychosocial treatments for child and adolescent disorders: Empirically based strategies for clinical practice (pp. 109-136). Washington, DC: American Psychological Association.

Lewinsohn, P. M., Hoberman, H. M., Teri, L., \& Hautzinger, M. (1985). An integrative theory of unipolar depression. In S. Reiss and R. R. Bootzin (Eds.), Theoretical issues in behavior therapy (pp. 313-359). New York: Academic Press.

Lewinsohn, P. M., Gotlib, I. H., \& Seeley, J. R. (1997). Depression-related psychosocial variables: Are they specific to depression in adolescents? Journal of Abnormal Psychology, 106, 365-375.

McCauley, E., Myers, E., Mitchell, J., Calderon, R., Schloredt, K., \& Treder, R. (1993). Depression in young people: Initial presentation and clinical course. Journal of the American Academy of Child and Adolescent Psychiatry, 32, 714-722.

Najavits, L. M. (1998). How to write a treatment manual. The Behavior Therapist, 21, 177-178.

Puig-Antich, J., Kaufman, J., Ryan, N. D., Williamson, D. E., Dahl, R. E., Lukens, E., Todak, 
G., Ambrosini, P., Rabinovich, H., \& Nelson, B. (1993). The psychosocial functioning and family environment of depressed adolescents. Journal of the American Academy of Child and Adolescent Psychiatry, 32, 244-253.

Reynolds, W. M., \& Coats, K. I. (1986). A comparison of cognitive-behavioral therapy and relaxation training for the treatment of depression in adolescents. Journal of Consulting and Clinical Psychology, 54, 653-660.

Renaud, J., Brent, D. A., Baugher, M., Birmaher, B., Kolko, D. J., \& Bridge, J. (1998). Rapid response to psychosocial treatment for adolescent depression: A two-year follow-up. Journal of the American Academy of Child and Adolescent Psychiatry, 37, 1184-1190.

RIESTER, A. E. (1994). Group psychotherapy for youth: Experiencing in the here and now. Journl of Child and Adolescent Group Psychotherapy, 4, 177-185.

Roberts, E. R., Lewinsohn, P. M., \& Seeley, J. R., (1991). Screening for adolescent depression: A comparison of depression scales. Journal of the American Academy of Child and Adolescent Psychiatry, 30, 58-66.

Roselló, J., \& Bernal, G. (1999). The efficacy of cognitive-behavioral and interpersonal treatments for depression in Puerto Rican adolescents. Journal of Consulting and Clinical Psychology, 67, 734-745.

Schwab-Stone, M. E., Shaffer, D., Dulcan, M. K., Jensen, P. S., Fisher, P., Bird, H. R., Goodman, S. H., Lahey, B. B., Lichtman, J. H., Canino, G., Rubio-Stipec, M., \& Rae, D. S. (1996). Criterion validity of the NIMH Diagnostic Interview Schedule for Children Version 2.3 (DISC-2.3). Journal of the American Academy of Child and Adolescent Psychiatry, 35, 878.

Shaffer, D., Fisher, P., Dulcan, M. K., Davies, M., Piacentini, J., Schwab-Stone, M. E., Lahey, B. B., Bourdon, K., Jensen, P. S., Bird, H. R., Canino, G., \& Regier, D. A. (1996). The NIMH diagnostic interview schedule for children version 2.3 (DISC-2.3): Description, acceptability, prevalence rates, and performance in the MECA study. Journal of the American Academy of Child and Adolescent Psychiatry, 35, 865-877.

Wood, A., Harrington, R., \& Moore, A. (1996). Controlled trial of a brief cognitive-behavioral intervention in adolescent patients with depressive disorders. Journal of Child Psychology and Psychiatry and Allied Disciplines, 37, 737-746.

YALOM, I. D. (1995). The theory and practice of group psychotherapy (4th ed.). New York: Basic Books. 